\title{
C ritérios semiquantitativos de análise da linfocintilografia em linfedema dos membros inferiores
}

\author{
Criteria for semi-quantitative analysis of lymphoscintigraphy in lower limb lymphedema
}

\author{
M arcelo Tatit Sapienza ${ }^{1}$, Irene Shimura Endo ${ }^{1}$, G iselle Christina Ferraro ${ }^{1}$, \\ M árcia G arrido M odesto Tavares ${ }^{1}$, G uilherme de $\mathrm{C}$ arvalho $\mathrm{C}$ ampos $\mathrm{N}$ eto ${ }^{1}$,

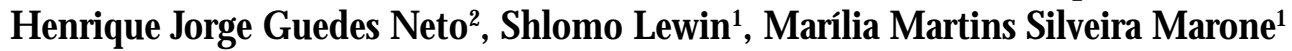

\section{Resumo}

O bjetivo: Avaliar se diferentes parâmetros de análise da linfocintilografia permitem a distinção entre membros com e sem linfedema em pacientes com quadro clínico de edema unilateral.

M étodos: Levantamento retrospectivo dos exames de 20 pacientes submetidos a linfocintilografia para investigação de edema unilateral de membros inferiores, divididos em pacientes com linfedema primário (grupo $1, n=7$ ) ou secundário pós-traumático (grupo 2, $n=$ 13). Foi realizada linfocintilografia após injeção subdérmica de soroalbumina humana marcada com tecnécio-99m nos pés, com imagens da região inguinal durante 15 min e imagens dos membros inferiores após 15 min e $1 \mathrm{~h}$. Os parâmetros analisados foram: tempo de aparecimento da cadeia inguinal, índice semiquantitativo (fluxo linfático, difusão intersticial, aspecto dos vasos, tempo de aparecimento em função do tempo da região inguinal. As medidas nos membros clinicamente acometidos foram comparadas aos membros sem linfedema em ambos os grupos.

Resultado: 0 tempo médio de aparecimento da cadeia inguinal $e$ 0 índice semiquantitativo foram significativamentemaiores nos membros com linfedema que nos membros sem linfedema quando considerados todos os pacientes, com maior retardo e índice semiquantitativo nos membros com linfedema do grupo 1 em relação aos do grupo 2.

C onclusão: A análise do tempo de aparecimento da cadeia inguinal e 0 índice semiquantitativo fornecem parâmetros objetivos de avaliação do linfedema que podem auxiliar na detecção e seguimento dos pacientes com linfedema.

Palavras-chave: Cintilografia, linfedema. e aspecto dos linfonodos inguinais) e inclinação da curva de atividade

\begin{abstract}
O bjective: To evaluate whether different parameters could be used in the analysis of lymphoscintigraphy to distinguish affected from unaffected limbs in patients with unilateral edema.

M ethods: Twenty patients who underwent lymphoscintigraphy for screening of unilateral lower limb edema were retrospectively assessed and divided into patients with primary lymphedema (group $1, n=7$ ) or secondary post-traumatic edema (group $2, n=13$ ). Technetium $99 \mathrm{~m}$-labeled human serum albumin was subdermally administered in the feet, followed by a dynamic lymphoscintigraphy of the pelvis during $15 \mathrm{~min}$ and static images of the lower limbs after $15 \mathrm{~min}$ and $1 \mathrm{~h}$. Analyzed parameters were detection time of groin activity, semi-quantitative index (lymph flow, interstitial diffusion, aspect of vessels, detection time and aspect of inguinal lymph nodes) and slope of groin time-activity curve. The parameters of clinically affected limbs were compared to those of the contralateral limbs in both groups.
\end{abstract}

Results: D etection time of groin activity and semi-quantitative index were significantly higher in affected limbs considering all patients, with a more severe delay and a higher semi-quantitativeindex in group 1 compared to group 2.

Conclusion: Analysis of detection time of groin activity and semiquantitative index can add objective parameters that can be used in the diagnosis and follow-up of patients with lymphedema.

Keywords: Scintigraphy, lymphedema.

\section{Introdução}

O sistema linfático tem como principal função a SP.

2. Disciplina de Cirurgia Vascular, Faculdade de Ciências Médicas, Santa Casa de São Paulo, São Paulo, SP.

Artigo submetido em 09.10.06, aceito em 19.12.06.

J Vasc Bras 2006;5(4):288-94.

Copyright $\odot 2006$ by Sociedade Brasileira de Angiologia e de Cirurgia Vascular. reabsorção de proteínas e líquidos do interstício, mantendo estável a composição extracelular. Além disso, os linfonodossão sítio importantedo sistema imune, neles ocorrendo a fagocitose, a apresentação de antígenos 
pelos macrófagos e a proliferação linfoplasmocitária. A linfopatia, com redução da função linfática e conseqüente déficit de drenagem do interstício, leva ao quadro de linfedema, caracterizado pelo aumento de volume e peso da região afetada, diminuição da função e alteração estética, podendo cursar com fibrose na evolução. Complicações como celulite e úlceras crônicas podem agravar 0 quadro inicial ${ }^{1}$.

O linfedema pode ser primário (de apresentação congênita, precoce ou tardia) ou secundário, sendo este associado principal mente a processos inflamatórios, neoplasias e trauma. Apesar da maioria dos casos de linfedema ser diagnosticada clinicamente, métodos de imagem podem ser úteis para excluir obstrução venosa e detectar ou confirmar o sítio linfático com disfunção ${ }^{1,2}$.

A linfocintilografia é um método de imagem de fácil realização e pouco invasivo, com baixo risco de complicações, podendo ser utilizada não só para a confirmação da suspeita clínica de linfedema, como também para o seguimento ou monitoração de resposta a medidas terapêuticas ${ }^{3,4}$. 0 método tem sensibilidade de 70 a $95 \%$ eespecificidade de 90 a $100 \%$ para linfedema, sendo proposto por diversos autores como exame inicial para afastar ou confirmar lesão linfática ${ }^{5-12}$.

Apesar dos bons resultados descritos, existem muitas variações da técnica de aquisição e interpretação da linfocintilografia. D entre os radiofármacos empregados, destacam-sea soroal bumina humana marcada com tecnécio-99m (SAH -99mT c, IPEN -SP), quereproduza dinâmica das proteínas na linfa, e os radiocolóides, também utilizados para pesquisa delinfonodo sentinela por serem fagocitados e retidos no linfonodo de drenagem ${ }^{5}$. As vias subcutânea e intradérmica $4,6,7,11,13-15$ podem ser empregadas, havendo progressão mais rápida com a administração mais superficial.

A busca de parâmetros quantitativos de análise, apesar de não prescindir da avaliação visual, visa facilitar euniformizar a interpretação dos estudos cintilográficos, possibilitando a detecção de pequenas al terações da função linfática. Além disso, a análise quantitativa pode facilitar a comparação entre estudos durante 0 seguimento ou após intervenções terapêuticas em um paciente.

0 presente trabalho teve por objetivo avaliar se diferentes parâmetros de análise da linfocintilografia permitem a distinção entre membros com e sem linfedema em pacientes com quadro clínico de edema unilateral.

\section{Métodos \\ Pacientes}

Foram avaliados retrospectivamente 20 pacientes com quadro de linfedema unilateral demembro inferior encaminhados ao Serviço de M edicina N uclear da U D D O -D iagnósticos M édicos entreos anos de $2000 \mathrm{e}$ 2004. O s pacientes foram separados em dois grupos: grupo 1 ( $n=7)$, com diagnóstico clínico de linfedema primário, egrupo 2 ( $n=13)$, com quadro delinfedema secundário a trauma ou cirurgia em membros inferiores. $\mathrm{N}$ ão foram incluídosno levantamento pacientesem investigação por quadro clínico de linfedema bilateral ou de membros superiores.

\section{Linfocintilografia}

Após avaliação clínica breve para confirmação do motivo do exame, os pacientes foram submetidos a linfocintilografia de membros inferiores. 0 estudo foi feito após injeção subdérmica de $1 \mathrm{mC}$ i deSAH - $99 \mathrm{mT}$ c no dorso dos pés, adjacente ao primeiro espaço interdigital. As imagens foram adquiridas em câmara cintilográfica modelo $\mathrm{H}$ elix (Elscint, $\mathrm{H}$ aifa).

Imediatamente após a injeção, adquiriu-seimagem anterior dos pés durante $10 \mathrm{~s}$ (para determinar a atividade injetada). A seguir, foi adquirida série de imagens na projeção anterior de bacia, com 30 s de duração por 15 min (Figura 1). D uranteestes 15 min, o pacientefoi orientado a movimentar os pés (rotação e flexo-extensão) a cada $3 \mathrm{~min}$. Ao término dos $15 \mathrm{~min}$, foram realizadas imagens anteriores e posteriores do abdome emembrosinferiores, repetidas no intervalo de $1 \mathrm{~h}$ após a injeção (Figura 2).

\section{Análise}

Registrou-se, para cada membro, o tempo de aparecimento da cadeia inguinal. Curvas de atividade em função do tempo foram obtidas após a delimitação visual de áreas de interesse nas cadeias inguinais, sendo calculada a inclinação das curvas ajustadas por regressão linear (expressa em aumento das contagens por segundo, corrigida pela atividade injetada).

A linfocintilografia foi também analisada por índice semiquantitativo (ISQ), modificado a partir do estudo de K leinhans ${ }^{16}$. 0 índice foi obtido pela 
soma de valores atribuídos a parâmetros da análise visual (fluxo linfático, refluxo dérmico, aspecto dos linfáticos e linfonodos, tempo de aparecimento dos linfonodos), com o mesmo peso na composição final do índice, conforme representado na Tabela 1. A modificação do índice foi introduzida porque a ad-

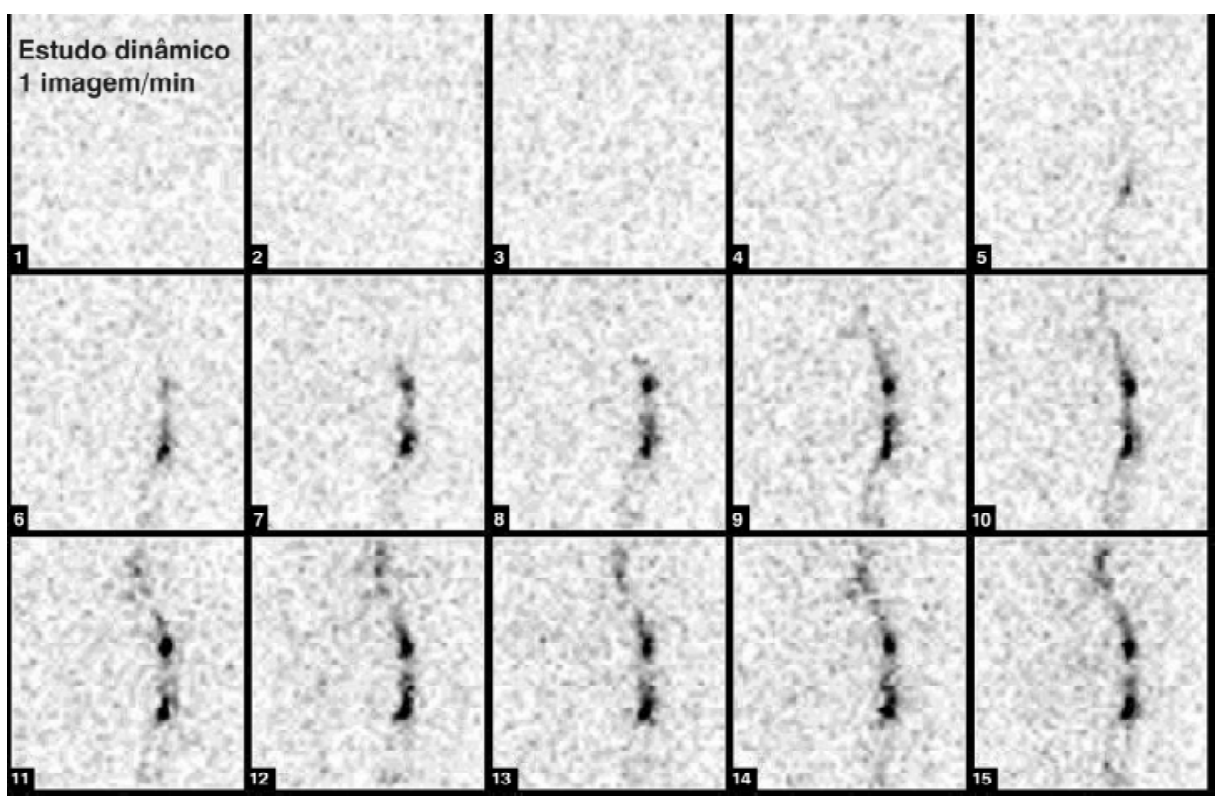

Figura 1 - Estudo dinâmico na incidência anterior de bacia em paciente com histórico de linfedema à direita, com aparecimento da cadeia inguinal esquerda após $5 \mathrm{~min}$ e ausência de progressão à direita
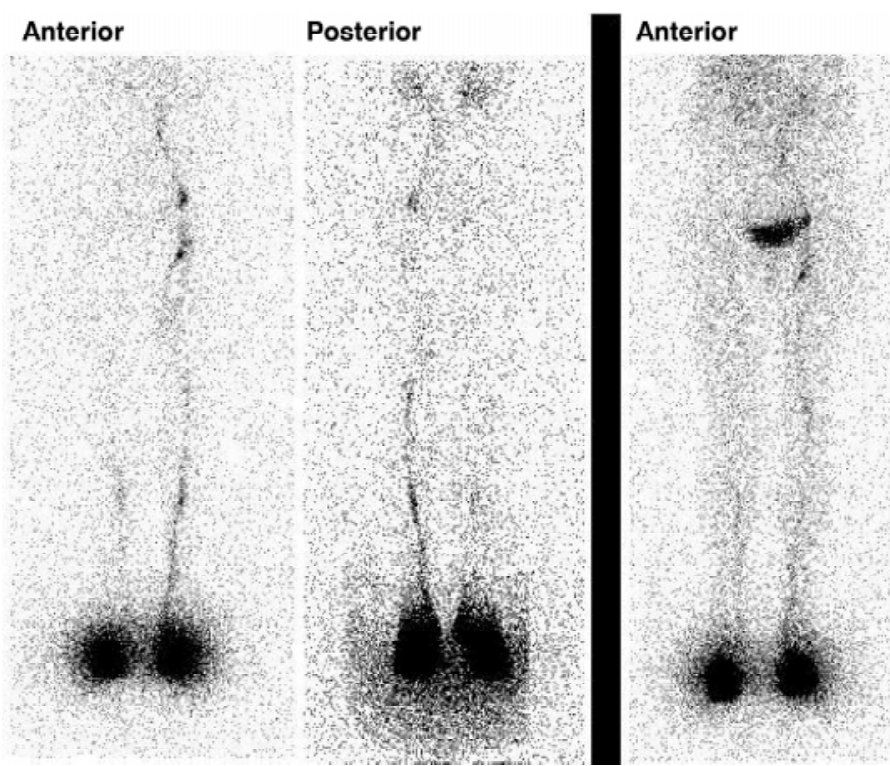

Posterior

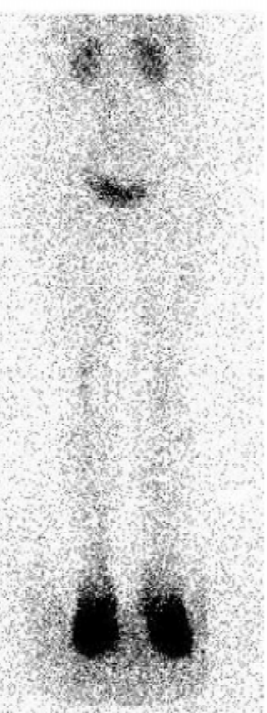

Figura 2 - I magens de membros inferiores, abdomee pelve realizadas 15 min e $1 \mathrm{~h}$ após a administração intradérmica da soroalbumina humana marcada com tecnécio-99m. N ota-se assimetria de ascensão, sem a identificação dos vasos e linfonodos à direita (índice semiquantitativo direito $=45$, esquerdo $=0$ ). M edicina N uclear da U D D O -D iagnósticos M édicos 
T abela 1 - V alores utilizados na elaboração do índice semiquantitativo

\begin{tabular}{|c|c|c|c|c|}
\hline \multirow{2}{*}{$\frac{\text { Parâmetro }}{\text { Fluxo linfático }}$} & \multicolumn{4}{|c|}{ Valor } \\
\hline & Fluxo normal $=0$ & $\begin{array}{l}\text { Redução discreta } \\
\text { a moderada }=3\end{array}$ & $\begin{array}{c}\text { Redução } \\
\text { acentuada }=5\end{array}$ & Fluxo ausente $=9$ \\
\hline D ifusão intersticial & $\begin{array}{c}\text { Ausente e } \\
\text { com fluxo =0 }\end{array}$ & $\begin{array}{c}\text { Discreta a } \\
\text { moderada }=3\end{array}$ & Acentuada $=5$ & $\begin{array}{c}\text { Ausente e } \\
\text { sem fluxo = }\end{array}$ \\
\hline Aspecto dos vasos linfáticos & $\mathrm{N}$ ormal $=0$ & Reduzidos $=3$ & Pouco visíveis = 5 & $\mathrm{~N}$ ão visíveis $=9$ \\
\hline Aspecto dos linfonodos & $\mathrm{N}$ ormal $=0$ & Reduzidos $=3$ & Pouco visíveis = 5 & $\mathrm{~N}$ ão visíveis $=9$ \\
\hline $\begin{array}{l}\text { Tempo de aparecimento } \\
\text { do linfonodo }\end{array}$ & Até $15 \min =0$ & Presente em $1 \mathrm{~h}=4,5$ & Ausente em $1 \mathrm{~h}=9$ & \\
\hline
\end{tabular}

ministração subdérmica da SAH - $99 \mathrm{mT}$ c leva a uma maior velocidade de progressão comparada ao enxofre coloidal-99mT c (diâmetro de 100 a $1.000 \mathrm{~nm}$ ) empregado no trabalho original deK leinhans, sendo considerado normal o "tempo de aparecimento do linfonodo" igual ou inferior a 15 min e acentuadamente retardado quando superior a $1 \mathrm{~h}$. A faixa possível de variação do ISQ total é de 0 a 45, sendo considerados normais os índices inferiores a 10.

Foi realizada a comparação en tre os três parâmetros (tempo de aparecimento, ISQ e inclinação da curva) dos membros com e sem linfedema no conjunto de pacientes estudados. $A$ análise comparativa entre 0 membro com esem linfedemadosmesmospacientesfoi feita pelo teste t de Student pareado, sendo considerados significativos val ores de $P<0,05$. Para comparação entre os membros com e sem linfedema dos grupos $1 \mathrm{e}$ 2, empregou-se 0 teste t não pareado.

\section{Resultados}

O s pacientes apresentavam idade de 10 a 74 anos (média $\pm D P=41 \pm 20$ anos), sendo seis do sexo masculino e 14 do sexo feminino. 0 s grupos 1 e 2 apresentaram diferença significativa de composição quanto à faixa etária, com idade de $22 \pm 12$ anos no grupo 1 e $51 \pm 15$ anos no grupo $2(P=0,0003)$.

0 tempo de aparecimento das cadeias inguinais, - ISQ e a inclinação das curvas de atividade inguinal dos membros com e sem linfedema nos pacientes de ambos os grupos são apresentados na T abela 2.
A análise conjunta dos pacientes dos grupos 1 e 2 mostrou tempo médio de aparecimento da cadeia inguinal significativamente maior nos membros com linfedema quenos membros sem linfedema $(P=0,02)$, assim como o ISQ ( $P<0,0001)$. N ão foi constatada diferença significativa na inclinação da curva inguinal entre os membros com e sem linfedema na análise conjunta de todos os pacientes ( $P=0,14)$.

A análisedosmembrossem linfedema não mostrou variação dos pacientes do grupo 1 em relação aos pacientes do grupo 2 quanto ao tempo deaparecimento da cadeia inguinal $(P=0,2)$, quanto ao ISQ $(P=0,17)$ ou quanto à inclinação da curva $(P=0,71)$.

A análise dos membros com linfedema evidenciou aparecimento da cadeia inguinal em tempo mais tardio $(P=0,02)$ e um maior ISQ $(P=0,03)$ dos pacientes do grupo 1 em relação aos do grupo 2, porém sem diferença significativa na inclinação da curva de atividade inguinal ( $P=0,17$ e 0,71).

Em três (23\%) pacientes do grupo 2, observouse difusão intersticial iniciada à distância do ponto deinjeção no membro com linfedema, acompanhada por uma identificação precoce da cadeia inguinal (em tempo inferior ao lado sem edema clínico). Este achado de refluxo dérmico à distância do sítio de injeção, não constatado em nenhum dos casos do grupo 1, é específico para linfedema secundário. Entre os sete pacientes com linfedema primário, dois $(29 \%)$ apresentaram tempo de aparecimento da cadeia inguinal de $60 \mathrm{~min}$. 
T abela 2 - Inclinação da curva de atividade inguinal versus tempo eíndice semiquantitativo

\begin{tabular}{|c|c|c|c|c|c|c|}
\hline \multirow[b]{2}{*}{ Paciente, idade (anos), sexo (F/M ) } & \multicolumn{2}{|c|}{ Tempo (min) } & \multicolumn{2}{|c|}{ Índice semiquantitativo } & \multicolumn{2}{|c|}{ Inclinação (cont/s) } \\
\hline & $\begin{array}{l}\text { Com } \\
\text { edema }\end{array}$ & $\begin{array}{l}\text { Sem } \\
\text { edema }\end{array}$ & $\begin{array}{l}\text { Com } \\
\text { edema }\end{array}$ & $\begin{array}{l}\text { Sem } \\
\text { edema }\end{array}$ & $\begin{array}{l}\text { Com } \\
\text { edema }\end{array}$ & $\begin{array}{l}\text { Sem } \\
\text { edema }\end{array}$ \\
\hline \multicolumn{7}{|l|}{ G rupo 1 - linfedema primário } \\
\hline$V A, 15, F$ & 60 & 3 & 18,5 & 0,0 & 0,0085 & 0,1164 \\
\hline $\mathrm{AO}, 33, \mathrm{M}$ & Ausente & 15 & 45,0 & 19,0 & 0,0059 & 0,0031 \\
\hline VLM S, $44, F$ & 60 & 60 & 36,5 & 17,5 & 0,0028 & 0,0014 \\
\hline$A O B, 22, F$ & 60 & 60 & 19,5 & 7,5 & 0,0140 & 0,0029 \\
\hline ACSG $, 15, M$ & 12 & 2 & 11,0 & 0,0 & 0,0551 & 1,7470 \\
\hline SN M , 15, M & 60 & 7 & 24,0 & 0,0 & 0,0198 & 0,1037 \\
\hline $\mathrm{JCS}, 10, \mathrm{~F}$ & Ausente & 1 & 29,0 & 0,0 & 0,0365 & 0,2708 \\
\hline
\end{tabular}

\section{G rupo 2 - linfedema pós-trauma/cirurgia}

M M M L, 38, F (trauma

direto há 3 meses)

$\begin{array}{llllll}60 & 4 & 10,5 & 0,0 & 0,0079 & 1,2090\end{array}$

CSM , 55, F (safenectomia

há 10 anos)

$\begin{array}{llllll}\text { Ausente } & 6 & 45,0 & 0,0 & 0,0224 & 0,2304\end{array}$

O SM , 69, M (cirurgia

articular joelho há 3 anos)

M CM P, 72, F (cirurgia

articular joelho há 3 meses)

M CC, 49 , $F$ (cirurgia de varizes

há 18 anos)

Ausente

0,0

0,0

0,9488

0,0677

AFS, 28, M (trauma há 17 anos)

2

15

6,0

0,0

0,0088

0,0333

M JT , 45, F (safenectomia há 7 meses)

12

9,0

6,0

0,0133

0,0332

60

15,5

0,0597

0,2499

5

3,0

0,0

0,1564

0,0911

M ILA, 54, F (safenectomia

bilateral há 14 e há 4 anos;

edema unilateral há 1 ano)

$M C M, 45, F$ (cirurgia de varizes

há 16 e 8 anos)

13

9,0

0,0

0,0077

0,1575

CDV , 57, $M$ (acidente de

automóvel há 6 semanas)

ITC, 74, F (safenectomia

medial da perna direita há 1 ano)

M FR, 54, F (cirurgia de varizes

há 1,3 anos)

8

8,0

0,0

0,0393

0,1584

EB, 30, F (cirurgia por sarcoma

inguinal há 3 anos)

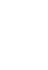

5

3,0

0,0

0,2448

0,505

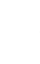

9

9

3,0

$0,0 \quad 0,0508$

0,1319

\section{Grupo 1 \\ M édia \\ D esvio padrão}

Grupo 2

M édia

D esvio padrão

5,0

6,0

0,0489

0,0085

T otal $(1+2)$

$M$ édia

D esvio padrão

Ausente

\section{0,4}

21,5

21,1

26,9

26,2

11,6

41,0

3,0

0,0046

0,0379

18,0

21,1

6,3

8,6

0,0204

0,3208

0,0190

0,6363

\section{8,1}

25,7
6,6

2,7

12,1

14,3
17,1

14,8
1,1

2,3

0,1241

0,2574

0,2241

0,3244

$\begin{array}{ll}11,7 & 17,1 \\ 16,9 & 14,8\end{array}$

2,9

5,8
0,0878

0,2111
0,2579

0,4434 


\section{D iscussão}

A linfocintilografia é um método de imagem de fácil realização e pouco invasivo, com baixo risco de complicações. Além da utilização diagnóstica na avaliação de edema de membros inferiores ou superiores ${ }^{17}$, pode ser feita para avaliação de resposta terapêutica ${ }^{14}$. A pesar dos bons resultados descritos na literatura, existem muitas variações da técnica de aquisição einterpretação da linfocintilografia. Em nosso serviço, optamos

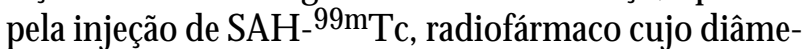
tro inferior a $80 \mathrm{~nm}^{11}$ é ideal para o estudo da função linfática, por não ser reabsorvida do interstício para os capilares sangüíneos ${ }^{15,18}$. A injeção é feita por via subdérmica, região rica em linfáticos iniciais e que permite rápida drenagem do radiofármaco.

A análise visual da linfocintilografia consiste no ponto principal da interpretação do estudo, devendo ser feita em conjunto com a análisedosdadosdehistória do paciente. $\mathrm{N}$ os casos de linfedema primário, há um retardo ou ausência de progressão linfática, com má caracterização dos vasos e dos linfonodos, podendo haver refluxo dérmico precoce a partir do ponto de injeção ${ }^{5}$. N os casos de linfedema secundário, os vasos podem ser proeminentes ou com colaterais, sendo um achado específico a presença de refluxo dérmico à distância do sítio de injeção, que indica a ruptura linfática.

Parâmetros deanálise semiquantitativos podem ser empregados de forma complementar à análise visual, para melhor caracterização de alterações discretas ou para a monitoração e avaliação terapêutica em estudos seqüenciais. D entre os parâmetros de análise visual, 0 tempo de identificação da cadeia inguinal destaca-se por sua objetividade $\mathrm{e}^{8,9,11}$. Os demais parâmetros de análise visual (relacionados à progressão linfática, características dos vasos e cadeias linfonodais ea presença de refluxo dérmico) também são incluídos no ISQ , o qual traduzem val ores numéricosos principaiscritérios da análise visual, tendo boa correlação com o quadro clínico delinfedema, com sensibilidadeeespecificidade de, respectivamente, 80 e $94 \%$ em estudo com 188 pacientes ${ }^{9,16}$ e possivelmentefacilitando a comparação de estudos no seguimento ou após intervenções terapêuticas. Em relação ao ISQ proposto por K leinhans, a única modificação neste trabalho foi o ajuste das notas paratemposmais precoces deaparecimento do linfonodo na cadeia inguinal, pois utilizamos a administração subdérmica deSAH, quetem ascensão mais rápida que a observada ao empregar colóides por via subcutânea.
Em nossa análise, o tempo de aparecimento da cadeia inguinal e o ISQ foram parâmetros adequados para a separação dos membros com e sem linfedema, além de mostrar um maior retardo no grupo 1, possivelmente decorrente do maior comprometimento da drenagem linfática nos pacientes com linfedema primário. U m achado interessantefoi aidentificação precoceda cadeia inguinal em três casos de linfedema pós-trauma, associadaa difusão intersticial à distânciado ponto deinjeção. Este achado sugere mecanismos compensatórios de drenagem linfática ativados em casos de linfedema por ruptura traumática.

O utros parâmetros numéricos são sugeridos para a análise da linfocintilografia, incluindo a avaliação da curva da atividade na região inguinal, que poderia refletir diretamente a progressão linfática do radiofármaco. Empregando um protocolo semelhante ao deste trabalho, Andrade encontrou grande variabilidade na taxa de contagens da região inguinal entre os pacientes ${ }^{15}$. Esta variação também é relatada por outros autores $^{11,19,20}$, e talvez seja, em parte, explicada por pequenas diferenças entre a atividade administrada em cada membro. Em nosso estudo, a análise da curva não possibilitou a distinção entre o membro com e sem linfedema, mesmo após correção pela atividade administrada, o quepossivelmenteindiquea interferência de outros fatores, como a variação na retenção do material em trânsito por cada linfonodo ou por toda a cadeia inguinal. D iversos autores propõem que, ao invés da chegada do radiofármaco na cadeia inguinal, o clareamento do ponto de injeção seria um mel hor parâmetro de quantificação ${ }^{19-22}$.

0 presente estudo apresenta limitações decorrentes do desenho retrospectivo, incluindo a dificuldade em estabelecer de forma padronizada o critério clínico de diagnóstico egravidade do quadro delinfedema primário ou secundário e a heterogeneidade dos grupos. A mais importante limitação do estudo deve-se à dificuldade de excluir ou avaliar com precisão as alterações venosas que podem contribuir para o quadro de edema epara a modificação dos padrões da cintilografia. Antes da realização do exame, foi regularmente realizada anamnese dirigida e questionado o histórico de trombose venosa profunda, porém não houve acesso direto ou registro sistemático dos dados de investigação vascular prévia. A comparação ao membro sem linfedema pode ser questionada pela possibilidade de acometimento subclínico, principalmentenos casos de linfedema primário. Porém, ressalta-se que o estudo não 
buscou estabelecer um valor "normal" para os parâmetros analisados, mas sim determinar se estes eram capazes de distinguir os membros com e sem linfedema clínico.

Em conclusão, apesar de a análise visual ser suficiente para a interpretação da linfocintilografia, a análise do tempo de aparecimento da cadeia inguinal e o ISQ fornecem parâmetros objetivos de avaliação do linfedema, que podem auxiliar na detecção e seguimento dos pacientes com linfedema.

\section{Referências}

1. Tiwari $A, C$ heng $K S$, Button $M, M$ yint $F, H$ amilton $G$. $D$ ifferential diagnosis, investigation, and current treatment of lower limb lymphedema. Arch Surg. 2003;138:152-61.

2. Guedes HJ, Andrade MFC. Diagnóstico e tratamento do linfedema periférico. Cir Vasc Angiol. 1996;12:62-5.

3. W eiss M, Baumeister RG, H ahn K. D ynamic lymph flow imaging in patients with oedema of the lower limb for evaluation of the functional outcome after autologous lymph vessel transplantation: an 8-year follow-up study. Eur J N ucl M ed M ol Imaging. 2003;30:202-6.

4. Kafejian-H addad AP, Perez JM, Castiglioni M L, M iranda J unior $F$, deFigueiredo LF. Lymphscintigraphic evaluation of manual lymphatic drainage for lower extremity lymphedema. Lymphology. 2006;39:41-8.

5. Scarsbrook AF, Ganeshan A, Bradley KM. Pearls and pitfalls of radionuclide imaging of the lymphatic system. Part 2: Evaluation of extremity lymphoedema. Br J Radiol. 2006; Epub ahead of print.

6. MCN eill GC, Witte $M H$, Witte $C L$, et al. Whole-body Iymphangioscintigraphy: preferred method for initial assessment of the peripheral lymphatic system. Radiology. 1989;172:495-502.

7. Rijke AM , Croft BY, Johnson RA, de Jongste AB, C ampsJA. $L$ ymphoscintigraphy and lymphedema of thelower extremities. J Nucl M ed. 1990;31:990-8.

8. Brautigam P, Foldi E, Schaiper I, Krause T, Vanscheidt W, $M$ oser $E$. Analysis of lymphatic drainage in various forms of leg edema using two compartment lymphoscintigraphy. Lymphology. 1998;31:43-55.

9. Cambria RA, Gloviczki $P, N$ aessens JM, Wahner $H W$. $\mathrm{N}$ oninvasive evaluation of the lymphatic system with lymphoscintigraphy: a prospective, semiquantitative analysis in 386 extremities. J V asc Surg. 1993;18:773-82.

10. zum W inkel K, H ermann H J. Scintigraphy of lymph nodes. Lymphology. 1977;10:107-14.

11. W eissleder $H$, W eissleder R. Lymphedema: evaluation of qualitative and quantitative lymphoscintigraphy in 238 patients. Radiology. 1988;167:729-35.
12. Gloviczki $P, C$ alcagno $D$, Schirger $A$, et al. N oninvasive evaluation of the swollen extremity: experiences with 190 lymphoscintigraphic examinations. J V asc Surg. 1989;9:683-9.

13. G omesSC. Linfocintilografiasuperficial demembrosinferiores com dextran-500-T c99m. Estudo em pacientes portadores de filariose edelinfopatia não filariótica[tese]. Recife: Centro de Ciências da Saúde Universidade Federal de Pernambuco; 1990.

14. PerezM D C J.C ompressão pneumáticaintermitenteseqüencial no linfedema dos membrosinferiores: avaliação cintilográfica com dextran marcado com tecnécio-99m [tese]. São Paulo: Escola Paulista de M edicina; 1996.

15. Andrade M FC. Avaliação da absorção e transporte linfático em pacientescom trombosevenosa profundaagudademembros inferiores através da linfocintilografia [tese]. São Paulo: Faculdade de M edicina da U niversidade de São Paulo; 1998.

16. Kleinhans E, Baumeister RG, Hahn D, Siuda S, Bull U, $M$ oser $E$. Evaluation of transport kinetics in lymphoscintigraphy: follow-up study in patients with transplanted lymphatic vessels. Eur J N ucl Med. 1985;10: 349-52.

17. Guedes-N eto HJ. Estudo linfocintilográfico qualitativo dos membros superiores de doentes com linfedema secundário a tratamento cirúrgico para o câncer demama [tese]. São Paulo: Faculdade de C iências M édicas da Santa C asa de São Paulo; 2002.

18. H ung JC, W iseman $G A, W$ ahner $H W$, M ullan BP, T aggart TR, Dunn WL. Filtered technetium-99m-sulfur colloid evaluated for lymphoscintigraphy. J N ucl Med. 1995;36: 1895-901.

19. Hollander W, Reilly P, Burrows BA. Lymphatic flow in human subjects as indicated by the disappearance of 1-131labeled albumin from the subcutaneous tissue. J Clin Invest. 1961;40:222-33.

20. Fernandez M J, D avies W T, O wen G M , T yler A. Lymphatic flow in humans as indicated by the clearance of 1251 -labeled albumin from the subcutaneous tissue of the leg. J Surg Res. 1983;35:101-4.

21. Carena M, Campini R, Z elaschi G, Rossi G, Aprile C, Paroni G. Quantitative Iymphoscintigraphy. Eur J Nucl Med. 1988;14:88-92.

22. Pain SJ, N icholas RS, Barber RW, et al. Q uantification of lymphatic function for investigation of lymphedema: depot clearance and rate of appearance of soluble macromoleculesin blood. J N ucl M ed. 2002;43:318-24.

\section{Correspondência:}

$M$ arcelo T atit Sapienza

Rua C onselheiro Brotero, $1486 / 1^{\circ}$ andar

CEP 01232-010 - São Paulo, SP

E-mail: mtsapienza@hotmail.com 